\title{
The P53 pathway: what questions remain to be explored?
}

\author{
AJ Levine ${ }^{\star, 1}$, W Hu${ }^{1}$ and $Z$ Feng ${ }^{1}$ \\ 1 Institute for Advanced Study and The Cancer Institute of New Jersey, \\ Princeton, NJ 08540, USA \\ * Corresponding author: AJ Levine, Institute for Advanced Study and The \\ Cancer Institute of New Jersey, Princeton, NJ 08540, USA. \\ Fax: + 1-609-924-7592; E-mail: alevine@ ias.edu
}

Received 22.12.05; revised 15.2.06; accepted 22.2.06; published online 24.3.06 Edited by A Braithwaite

\begin{abstract}
The p53 pathway is composed of hundreds of genes and their products that respond to a wide variety of stress signals. These responses to stress include apoptosis, cellular senescence or cell cycle arrest. In addition the p53-regulated genes produce proteins that communicate these stress signals to adjacent cells, prevent and repair damaged DNA and create feedback loops that enhance or attenuate p53 activity and communicate with other signal transduction pathways. Many questions remain to be explored in our understanding of how this network of genes plays a role in protection from cancers, therapy and integrating the homeostatic mechanisms of stress management and fidelity in a cell and organism. The goal of this chapter is to elucidate some of those questions and suggest new directions for this area of research.

Cell Death and Differentiation (2006) 13, 1027-1036.

doi:10.1038/sj.cdd.4401910; published online 24 March 2006
\end{abstract}

Keywords: The p53 pathway; feedback loops; apoptosis; stress responses; DNA damage

Abbreviations: MDM-2, murine double minute 2; COP-1, coat protein complex 1

\section{Introduction}

\section{The goals for this chapter}

The p53 protein was first described in $1979^{1-4}$ and since that time there have been more than 35000 papers published on this topic. The description of this protein and its gene has changed from a virus-associated tumor antigen to an oncogene to a tumor suppressor gene. ${ }^{5}$ The major functions of the p53 protein have been elucidated and reasonable explanations are in hand that describe why it is an important tumor suppressor gene in humans and animals. ${ }^{6}$ This progress has been reported at 12 different meetings dedicated solely to research with the p53 protein, which have been held every other year starting in 1981, with the most recent meeting being held in New Zealand (2004) and the next one will be in New York City, USA (2006). As each meeting results in hundreds of presentations and posters they provide the latest concepts of how the p53 protein functions and what its many roles are in the cell and the organism. From this a consensus forms and new questions emerge that change the concepts, ideas and directions of this area of research. That is just what happened at the last meeting and the goal of this chapter is then to help guide and formulate the questions that remain to be explored over the next years. Some of these questions address very specific observations and are quite narrow, but important to answer. Other questions are global and address large conceptual issues in the field. The format of this chapter will be to describe the functioning of the p53 pathway and at each specific step to identify those narrow and focused questions that require the attention of experimentalists and need answers. By asking these questions we may uncover contradictions in the literature, point out results that remain unclear or bring up issues that remain to be tested and are unresolved. As we move through the descriptions of each step in the p53 pathway and note these focused issues they will collectively point to holes in our understanding and lead to the bigger issues. In the final section of this chapter the larger or global questions can be explored in the context of understanding the details of the p53 network and its functions.

The p53 pathway may conveniently be divided up into five parts (Figures 1 and 2); (1) The input signals that trigger or induce the network into a functional state. (2) The upstream mediators that detect and interpret those signals that initiate the functional pathway and relay the inputs to the p53 protein or molecules that most immediately (in minutes to an hour) regulate the concentration and activity of the p53 protein. (3) The core set of proteins, including the $\mathrm{p} 53$ protein itself, which regulates p53 activity and function. (4) The downstream events which are composed of a set of genes and their proteins that are regulated by the $\mathrm{p} 53$ protein, most commonly by transcriptional activation but in some cases by proteinprotein interactions. (5) The cellular outputs of these downstream events which include cell cycle arrest, cellular senescence or apoptosis and often result in extensive communication with other signal transduction pathways in the cell. Employing this format we can identify the open questions that remain to be explored. Then we can turn our attention to more global questions about these processes in a larger context of the cell and organism.

\section{The P53 Pathway}

\section{The input signals that trigger or induce the p53 pathway}

The p53 protein and its signal transduction pathway are composed of a set of genes and their protein products that are 
Activation of p53

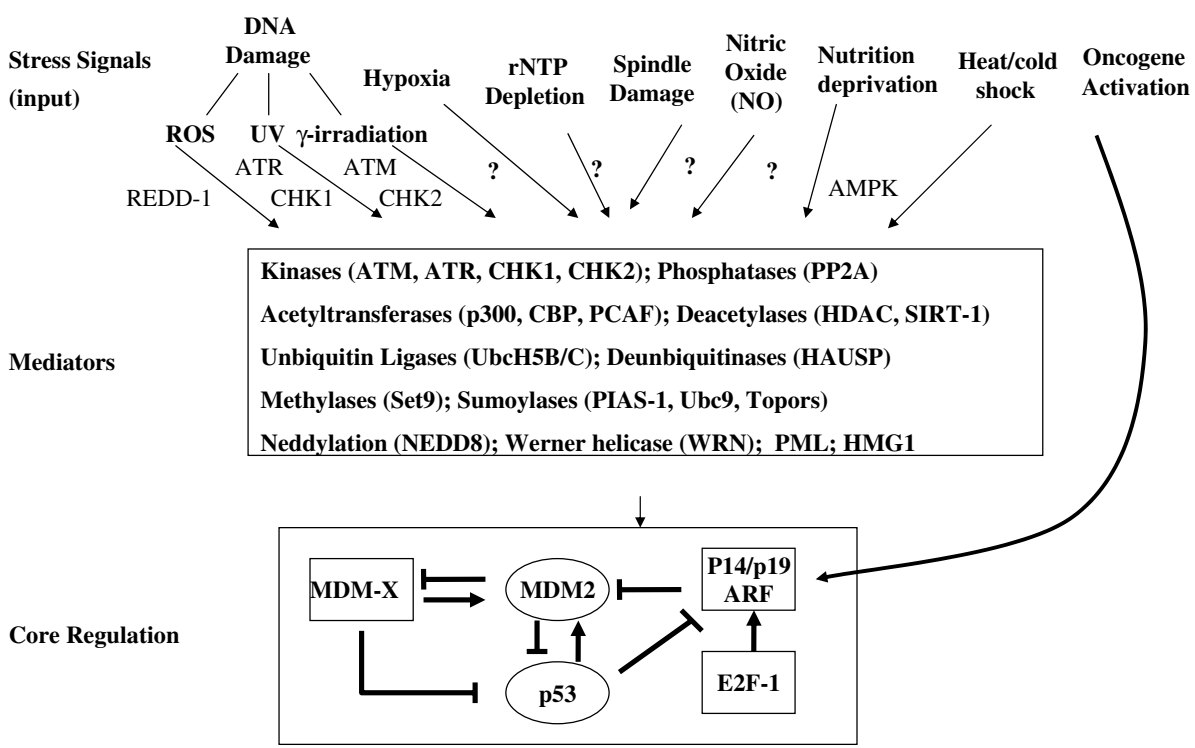

Figure 1 The input signals, mediators and core p53 functions of the p53 pathway. Several types of stress signals are detected by the cell and communicated to the p53 protein and its core constituents by the mediators. Several stress signals result in the degradation of the MDM-2 protein and the increase in the levels and activity of the p53 protein. The MDM-2 protein levels are then restored via the p53-mediated transcription of the MDM-2 gene

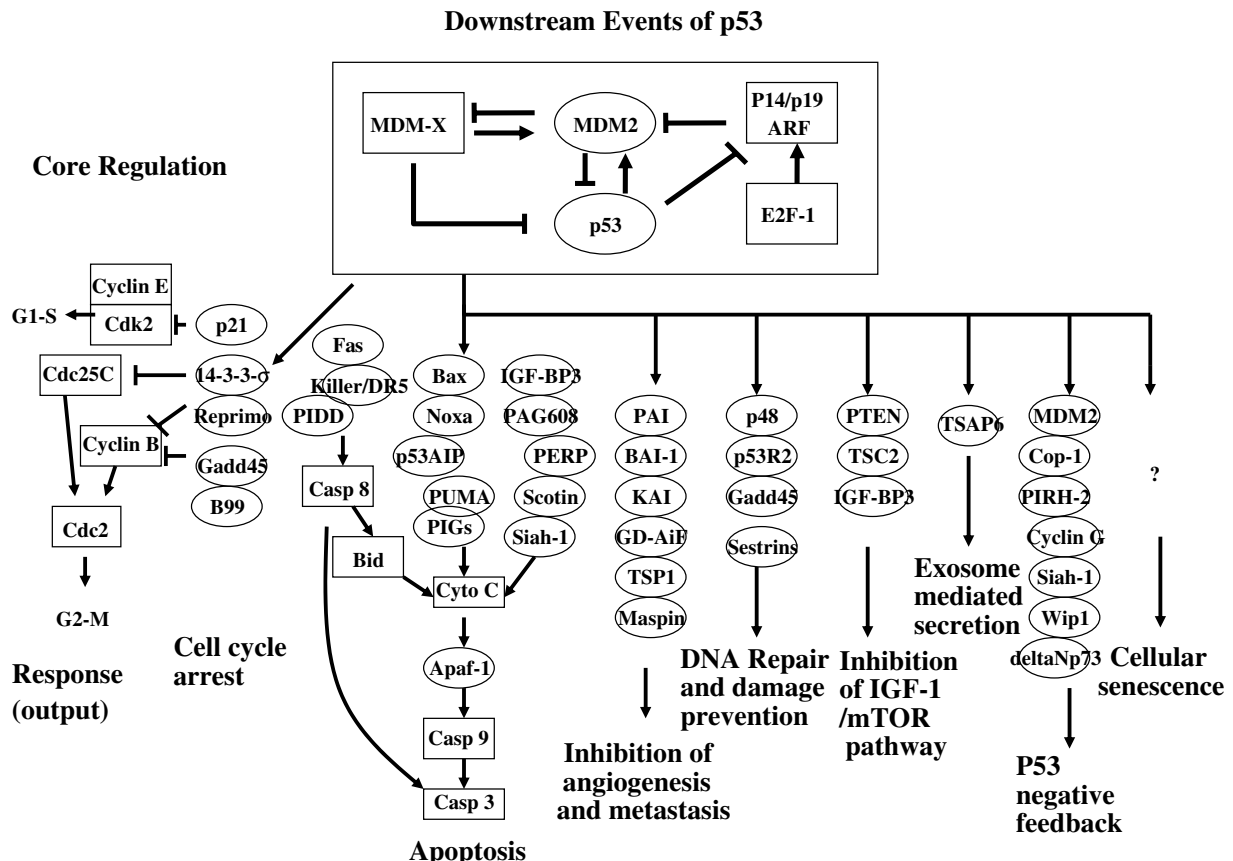

Figure 2 The core p53 functions, the downstream genes regulated by p53 and the outputs of the p53 pathway. The p53 protein is employed as a transcriptional activator of p53-regulated genes. This results in three major outputs; cell cycle arrest, cellular senescence or apoptosis. Other p53-regulated gene functions communicate with adjacent cells, repair the damaged DNA or set up positive and negative feedback loops that enhance or attenuate the functions of the p53 protein and integrate these stress responses with other signal transduction pathways

designed to respond to a wide variety of intrinsic and extrinsic stress signals. These stress signals all impact upon the cellular homeostatic mechanisms that monitor and control the fidelity of DNA replication, chromosome segregation and cell division. ${ }^{7}$ Among the stresses that activate the $\mathrm{p} 53$ protein are damage to the integrity of DNA in a cell. There are many physical and chemical causes of DNA damage including gamma or UV irradiation, alkylation of bases, DNA crosslinking, depurination of DNA, alteration of the deoxyribose sugar moiety, reaction with oxidative free radicals and more. 
Each of these types of DNA damage is different and each is detected by a different set of proteins and then repaired by different enzymes or activities that reverse the damage in diverse ways. There are multiple DNA damage detection and repair systems in the cell but every type of DNA damage is reported to the p53 protein and its pathway. ${ }^{8-10}$ The duplication processes of DNA often make mistakes (i.e. mismatch errors) that are in the main edited and repaired, but when lesions remain they too can be detected by p53. In all of these cases the p53 pathway functions to respond to errors (a check on the fidelity of these processes) and eliminates those cells with such mistakes. As cells divide and the telomeres of chromosomes get shorter they reach a critical size that can result in an enhanced rate of translocations or abnormal recombination events. The presence of these shorter telomeres in a cell are reported to the p53 protein resulting in a p53 response which in turn limits abnormal chromosome translocations. In addition to DNA damage several cellular deprivations activate the p53 response such as hypoxia, glucose starvation or the depletion of ribonucleoside triphosphate pools in the cell. When ribosome biogenesis is stopped or falls below a critical level the p53 protein is alerted and activated. Agents that damage the cell spindle and result in faulty chromosome segregation activate p53. Both heat and cold shock as well as the altered or denatured protein response in cells communicate with p53 by activating it. Nitric oxide, which is often associated with infections and inflammation activate the p53 protein and its response. All of these stresses will result in a loss of fidelity in the cellular duplication process and as such their communication with the p53 pathway acts as a check point to prevent abnormal clones of cells from arising. The mutational activation of some oncogenes also result in the sensitization of the p53 response and can often change the downstream output of the p53 pathway from cell cycle arrest to apoptosis. This very large number of diverse cellular stress signals that all feed into a central and single node to monitor and respond to those stresses may be thought of as a poor design of a system that is too vulnerable to the loss of a single central node. ${ }^{7}$ Why don't different stress signals act upon different protein checkpoints? Why is the pathway not duplicated or backed up so as to make it more robust and less vulnerable to the mutational damage of the p53 gene? The observation that demonstrates the unique and central function of the p53 protein in this stress network is that the p53 gene is mutated about $50 \%$ of the time in a wide variety of cancers. ${ }^{11}$ In a number of other cancers additional genes in the network that encode proteins that act upon the p53 functions are mutated. Thus, most cancers appear to select for a loss of function of the $\mathrm{p} 53$ pathway. ${ }^{12}$ The most common answer to why evolution has designed the network to have a single central node that receives inputs from a wide variety of stress signals is that one entity or protein can act as the most efficient integrator of information about the different types of stress that act upon the cell. Multiple proteins receiving multiple input signals would have to have a much more complicated communication system to integrate information about the environmental stresses. Because all of these stresses lead to a lack of fidelity in the duplication process, and that could lead to disease, the p53 protein is a central player in the go-no go decision of a cell to grow or reproduce. The question remains; is this explanation a correct interpretation of the network architecture or is there a lot more to learn so as to answer this enigma?

What other stress signals can induce a p53 response? What more remains to be elucidated in the type or nature of the input signals? Can psychological stress, in the form of depression or nervous system activity activate the p53 protein? Do some hormonal changes result in the activation of the p53 protein? What nutritional changes, in addition to glucose deprivation, ${ }^{13,14}$ can induce a 053 response? It will be important to fill in those inputs to the p53 pathway over the next few years. In some cases (i.e. psychological stress) it will be a real challenge to design experiments to prove these points. Most of the experiments in the past that test for the activation of the p53 protein and therefore define an input signal for this pathway have been carried out in cell culture. In order to better define environmental stress we will need to carry out experiments (like testing psychological stress) in the whole organism taking into account cell type and tissue specificity as well as the variable of time (age) and developmental stage.

Orthologues of the p53 gene have been identified in worms, flies, mice and humans and it will clearly be instructive to elucidate a comparative genomic and network analysis and follow changes in p53 function over evolutionary time scales. Similarly two homologues of the p53 gene, p63 and p73, have been identified ${ }^{15,16}$ and it will be important to complete the characterizations of their functions, their possible role in diseases and their impact upon the $\mathrm{p} 53$ gene and its protein. ${ }^{17}$ Do $p 63$ or $p 73$ respond to stress signals and if so which ones? P73 is phosphorylated by the Abl kinase after DNA damage and $p 73$ can induce cellular apoptosis. There is a great deal to be learned about these functions in the cell and organism.

\section{The upstream mediators of the P53 response}

In response to an input stress signal the p53 protein is activated. We can define activation experimentally as an increase in the concentration of the p53 protein and an increased activity of a p53 protein for the transcription of a set of genes that have a p53 DNA response element ${ }^{18}$ and are transcriptionally regulated by the p53 protein. The levels of the p53 protein are predominantly regulated by it's proteolytic turn over. The p53 protein has a short half-life of $6-20 \mathrm{~min}$ in several cell types and the ubiquitin ligase that confers this short half-life is the MDM-2 protein. Recently, two other ubiquitin ligases were shown to act upon the p53 protein, COP-1 and PIRH-2. ${ }^{19,20}$ Why is this redundancy employed? What are the roles of these enzymes for the many diverse stress inputs, in different tissue or cell types or at the different times in development of an organism? After some types of DNA damage (gamma radiation) the MDM-2 protein is autopoly-ubiquitinated resulting in its degradation and an associated increase in $p 53$ levels and activity. ${ }^{21}$ While this observation helps to explain why the p53 protein has a longer half-life after some types of DNA damage, this mechanism is not observed in all types of DNA damage or stress signals. Indeed given the large number of stress signals employed as an input to the pathway we know almost nothing about how these diverse inputs are communicated to the p53 protein. 
That is a big challenge for future experiments. We do know that the p53 and MDM-2 proteins are extensively modified after a stress signal. ${ }^{22}$ The $\mathrm{p} 53$ protein is phosphorylated on a large number of serine and threonine residues by many different protein kinases. It is acetylated by histone acetyltransferases, methylated by methylases, ubiquitinated, summolated and neddylated (at epsilon amino groups of lysine at the carboxy-terminus of the $\mathrm{p} 53$ protein).${ }^{23-25}$ In some cases it is clear that this is part of the process that mediates the response from DNA damage to the p53 protein. For example, gamma radiation activates the ATM protein kinase that then phosphorylates MDM-2 and p53 (probably through a CHK kinase) as well as participating in the DNA repair process. In spite of these clear observations, mutations in many of the p53 protein serine and threonine residues that then block p53 phosphorylation events still result in fairly normal p53 activation and function. Similarly, the use of Nutlin (a drug that blocks p53-MDM-2 binding) activates p53 normally with no phosphorylation events. ${ }^{26}$ Changes of the lysines in p53 protein, that are normally acetylated or modified by various peptides, to arginine residues that cannot be modified resulted in normal p53 responses in mice with these mutant proteins. These types of experiments indicate that the protein modifications of the p53 protein after a stress input are not essential for the activation of the $\mathrm{p} 53$ protein (or are heavily backed up by other functions not eliminated in these experiments). This brings up the question; what are the functions, if any, of the protein modifications in p53 or the MDM-2 protein after exposure to a stress? Most people believe there is a functional role for these modifications. First of all the extent and nature of these protein alterations of the p53 protein differs with different types of inputs. Thus, these protein modifications form a chemical code that could inform the p53 protein and the cell about the type of stress that is occurring. Second the p53-regulated transcriptional output after different stresses is different, and these protein modifications could well dictate which p53-responsive genes are transcribed by the cell based upon the specific protein modifications of the p53 protein. Do p53 protein modifications determine the genes which are transcriptionally regulated by that p53 protein? These ideas need to be tested. P53 protein interactions with other cellular proteins (which may be initiated by protein modifications) impact upon gene selection and the nature of the downstream events. ${ }^{27,28}$ Just what protein modifications of p53 and MDM-2 accomplish in a cell remains a big question? In particular, the protein modifications and protein-protein interactions (with p19 ARF, cyclin G, etc.) of MDM-2 remain to be explored in more detail.

One of the most interesting methods of activating p53 results from the mutational inactivation of a tumor suppressor gene like the retinoblastoma protein (which activates the E2F-1 transcription factor) or the APC tumor suppressor (which activates the beta catinin-TCF4 transcription factor) or the mutational activation of oncogenes like myc or Ras. The activities of the transcription factors that result from mutations in these genes can transcribe the ARF gene and the ARF protein then binds to the MDM-2 protein and inhibits its activity as a ubiquitin ligase. This raises the p53 levels in a cell and helps to bring about a p53 response or output that protects the organism from the development of cancers. ${ }^{29,30}$ This is an excellent example of how diverse signal transduction pathways in a cell communicate and create feedback loops that contribute to cellular homeostasis. Just who the other players or modifiers of this circuit are remains to be explored? There are some suggestions that ARF has additional functions and may be regulated in several ways, and these questions remain a high priority for the field.

\section{The core control of P53}

Once activated the p53 protein transcribes a number of genes. Among these is the MDM-2 gene (and the COP-1 and $\mathrm{PIRH}-2$ genes) which is the major negative regulator of p53 in the cell. ${ }^{12}$ The transcriptional regulation of MDM-2 by p53 is a slow step (hours) but once the MDM-2 protein is produced it binds to p53 (inhibiting its activity as a transcription factor) and ubiquitinates it enhancing its proteolytic breakdown. This occurs as a rapid step. This auto-regulatory loop produces an MDM-2-p53 oscillator composed of changing protein levels of p53 and MDM-2, out of phase with one another, in the cell. ${ }^{31,32}$ When p53 levels rise this increases MDM-2 levels which in turn lower p53 levels, resulting in less MDM-2, etc. These oscillations have been observed in vivo to be variable from cell to cell but they can last a long time and the number of oscillations is roughly proportional to the input dose of radiation. ${ }^{32}$ Does transcriptional activation of the downstream genes depend upon this oscillator or does it optimize the output of the pathway? Just what the functions of such oscillations are, if any, and the factors that can modify these oscillations remain to be determined? Do COP-1 and PIRH-2 participate in this process? The reason that the field has focused upon MDM-2 as the major regulator of p53 is that it was found first ${ }^{33,34}$ and the gene knockout of MDM- 2 in mice is lethal early in development, just after implantation. ${ }^{35}$ This could be due to an hypoxia response of the p53 protein in the absence of MDM-2, so that p53 activity is uncontrolled. This idea needs to be tested. The double knockout mouse, with no MDM-2 or p53 genes, is viable proving that MDM-2 acts to block p53-mediated cell death early in development (is this due to hypoxia?). This also demonstrates that $\mathrm{PIRH}-2$ and COP-1 do not back up MDM-2, at least early in development. Now similar experiments should be done with COP-1 and PIRH-2 knockouts.

The gene products that regulate MDM-2 (and p53) levels and activities will need to be better characterized. The MDM-X (or MDM-4) gene was identified because it is related to the MDM-2 gene in its DNA and protein sequence. A knockout of the MDM-X gene in mice is lethal and a MDM-X and p53 double knockout is viable. ${ }^{36}$ Thus, MDM-X is as important as MDM-2 in regulating $\mathrm{p53}$ in vivo. It now appears that MDM-X can negatively regulate $p 53$ directly and positively regulates MDM-2. After a stress signal, the poly-ubiquitination of MDM2 results in the degradation of MDM-X and MDM-2, increasing p53 levels and activity. Just how this is accomplished (via protein modification of MDM-X or 2 , or protein-protein interaction) remains to be studied. The activities that modify MDM-X and MDM-2 (cyclin G-PP2A, ARF and its activators and HAUSP) act only on MDM-2 and remain to be explored in more detail. High levels of p53 appear to repress the transcription of the ARF gene and the p53 knockout mouse 
(no p53 protein) has much higher levels of ARF mRNA and protein than a wild-type mouse. How the p53 transcription factor acts to repress transcription remains a mystery and is in need of study.

Several studies have suggested additional levels of the control of the p53 protein, at the transcriptional and at the translational level, which should now form the basis for future research. In addition, the p53 and MDM2 proteins can shuttle between the nucleus and the cytoplasm which undoubtedly changes their activity and regulation. ${ }^{37,38}$ Just what factors control this cellular localization and therefore can impact upon p53 function remain to be explored. The AKT-1 kinase can phosphorylate the MDM-2 protein increasing its activity and sending it into the nucleus of the cell. ${ }^{39,40}$ Cyclin $\mathrm{G}$, a p53-regulated gene product, combines with the catalytic subunit of the PP2A phosphatase to remove a phosphate residue from the MDM-2 protein and increase its activity. ${ }^{41}$ These two modifiers of the MDM-2 protein form feedback loops that connect the core p53 activity to other signal transduction pathways as well as regulate the core functions of the network. Indeed there are at least 10 negative or positive feedback loops that start with a p53-regulated gene product and result in increasing or decreasing the activity of p53. ${ }^{12}$ These feedback loops connect the core p53 activities to other signal transduction pathways. When a positive feedback loop is activated it results in apoptosis and cell death, a negative feedback loop attenuates the p53 pathway. Do these loops act in all cell types? How are they regulated or triggered? What is the impact of the activation of another signal transduction pathway upon the core p53 regulation? A good example of this is the starvation of a cell for glucose. This engages the mTOR pathway. The absence of glucose activates the LKB kinase and the AMP kinase. The AMP kinase phosphorylates the TSC-2 protein in a TSC-1/2 complex. TSC-1/2 is a GAP that inactivates a G-protein, RHEB, and RHEB activity controls the kinase activity of mTOR. Thus, glucose starvation inactivates mTOR which leads to the activation of autophagy, a catabolic process that breaks down proteins, fats and carbohydrates into their momomeric components, supplying endogenous nutrients to the starved cell. While this proceeds the AMP-kinase also phosphorylates serine-15 on the p53 protein. ${ }^{13,14}$ In normal cells this does not activate the p53 protein but in transformed cells this is a proapoptotic event. P53 serine-15 phosphorylation is a precursor for other phosphorylation events for the p53 protein. ${ }^{22}$ When glucose becomes available and mTOR reactivates, mTOR activates a subunit of PP2A which can then remove the phosphate residue from p53 serine-15. ${ }^{42}$ This restores p53 to its original state and attenuates the feedback loop.

As there are two distinct transcriptional start sites for the p53 mRNA and several alternative splicing patterns of this mRNA there are nine different isoforms of the p53 protein that have been detected. The amino-terminus of the p53 protein has two distinct transcriptional activator regions of the protein and one or both can be present. The carboxy-terminus of the p53 protein has a basic domain which can be spliced out or replaced by another amino-acid sequence. All combinations of these alternative protein isoforms have been observed ${ }^{43}$ but many questions remain. Are these forms found preferen- tially in different cell or tissue types or at different stages of development? What are the functions of each alternative domain and the resultant protein? Does a protein with its DNA-binding domain intact but without a transactivation domain act as a repressor? How do the three alternative forms of the carboxy-terminus alter the functions of the p53 protein? Recently, one of these isoforms has been shown to have a distinct and critical function in the development of the gut of zebra fish. ${ }^{44}$ There is now clearly more to learn.

\section{The downstream events in the p53 pathway}

Once p53 is activated in response to a stress signal it gains the ability (and increased concentration) to bind to p53-responsive DNA sequence elements in the genome. The consensus DNA sequence for p53 binding is RRRCWWGYYY, where $R$ is a purine, $W$ is $A$ or $T$ and $Y$ is a pyrimidine. A p53-responsive element is composed of two of these 10 base paired sequences, separated by a spacer of $0-21$ base pairs, and the sequences are often located $5^{\prime}$ to the gene or in the first or second intron of the gene regulated by p53. ${ }^{45}$ It is a challenge to identify all of the p53-responsive sequences in a genome because degenerate sequences also function in a p53-dependent fashion. An algorithm has been described ${ }^{18}$ that has successfully identified novel p53responsive genes. ${ }^{13,46}$ It has become clear that different types of stress signals as inputs result in different genes being transcribed under p53 control. ${ }^{47}$ In addition stress signals received by different cell or tissue types also produce different transcriptional programs regulated by the p53 protein. We do not understand what mediates these different regulatory responses. Both p53 protein modifications and proteinprotein interactions could play a role as well as differences in the DNA sequences of response elements. In addition, there are large differences in both the amounts of mRNA and proteins produced by different p53-regulated genes and in the kinetics of synthesis after a p53-activation event. Why this is so remains unclear. There appear to be some p53-regulated genes that are transcribed in response to many different types of stress signals and in all tissues responding to the stress (p21, cyclin G, MDM-2, GADD-45) and others that are either stress- or tissue specific (PTEN, TSC-2). What regulates these differences remains unclear? The functions of the p53response genes fall into several categories. A set of genes are clearly involved in cell cycle arrest (p21, 14-3-3 sigma, GADD45). A second set of p53-regulated genes are involved in apoptosis. These can be divided into the intrinsic and extrinsic apoptotic pathways. In the extrinsic pathway p53 regulates Fas production (a secreted protein), as well as killer/DR5, the trail receptor and a membrane protein. These proteins, along with PIDD, activate caspase 8 and Bid to release cytochrome $c$ which acts with APAF-1 (a p53-regulated gene) to activate caspase 9 and then 3 resulting in apoptosis. The intrinsic pathway is populated with many p53-regulated genes of which bax, noxa and puma may work in different cell types to promote cytochrome $c$ release. Several other p53-regulated genes have been implicated in the enhancement of apoptosis (Perp, scotin, some PIGS, p53 AIP) but their mechanism of action remain to be elucidated. Elucidating the details of how p53 initiates apoptosis employing its transcriptional program 
is a high priority for the field. More recently several groups ${ }^{48,49}$ have provided good evidence that the p53 protein itself can move out of the nucleus and act upon the mitochondria or proteins in the mitochondria $(\mathrm{BCl}-2, \mathrm{BCL}-\mathrm{XL})$ to promote cytochome $c$ release and apoptosis. A large challenge remains to prove these mechanisms are functional in an animal and augment or replace, under some circumstances, the transcriptional-mediated apoptosis. This is presently a confusing and challenging area of research and needs to be resolved with a consensus being formed in the field. The third output of the p53 response is senescence of cells. There are some suggestions that senescence is as important as apoptosis in mediating the tumor suppressor functions of p53. In spite of this we have no idea which p53-regulated genes contribute to senescence and what gene products populate the pathway to cellular senescence. As normal cells divide and age in culture and telomeres shorten, p53 levels rise, but what transcriptional program this triggers remains to be elucidated? Mice with an overactive p53 protein, where the threshold for stress responses is lowered, die prematurely and have compromised stem cell capabilities (senescence of the stem cells). ${ }^{50}$ These mice are more resistant to developing cancers initiated with carcinogens. These mice also die at younger ages than wild-type mice with a normal p53 protein. We need to design new experimental approaches to explore these questions of cancer resistance and longevity in more detail. These experiments also introduce the question; what are the functions, if any, of the p53 protein in regulating longevity in animals and cells? The next few years will see these questions addressed in worms, flies, mice and even humans.

While cell cycle arrest, apoptosis and senescence are traditionally thought of as the major outputs of the p53 pathway several other p53-responsive genes are beginning to define additional functions of the p53 pathway. The p48 protein and p53R2 subunit of ribonucleotide reductase are p53-responsive genes that aid in DNA repair. The sestrins are a set of p53-regulated genes that counter the presence of reactive oxygen species in the cell. ${ }^{51}$ Clearly some p53 pathway functions help to protect the cell from exogenous or endogenous stresses while others enhance the cellular repair process. A second p53-regulated function is to communicate with cells in its environment that a cell has DNA damage or senses a stress signal. A number of p53-responsive or p53regulated genes produce secreted proteins. These proteins fall into several functional categories. The IGF-BP3 binds the IGF-1 hormone and prevents it from activating a growth response signal transduction pathway (IGF-1, IGF-1R, PI3 K, PDK, AKT-1, forkhead transcription factors). ${ }^{12,52}$ In this case p53 is negatively regulating cell growth, mitogen signaling and preventing cell division in surrounding cells after a stress signal. The p53-regulated and -secreted genes PAI and maspin are protease inhibitors that result in alterations to the extracellular matrix and cell surface. ${ }^{53,54}$ Similarly the p53regulated thrombospondin gene produces a secreted protein that alters the cellular matrix and is anti-angiogenic. ${ }^{55}$ Secreted proteins permit communication between cells. More recently the p53-regulated TSAP-6 gene was shown to enhance the rate of exosome production from cells undergoing a p53 response to stress. ${ }^{56}$ Exosomes also can communicate with other cells both in the immune system and cells in its own vicinity. Cells in stress will want to coordinate their responses with the surrounding cell layer in vivo and this is a new field opening up to future research efforts. Finally, a large number of p53-responsive genes (p21, WIP-1, SIAH-1, PTEN, TSC-2, IGF-BP-3, cyclin G, p73delta $\mathrm{N}, \mathrm{MDM}-2$, COP-1 and $\mathrm{PIRH}-2$ ) initiate positive or negative feedback loops with the p53 protein and the core gene products of the pathway. ${ }^{12}$ This results in the integration of a stress signal with other pathways in the cell that regulate cell growth, division or cell death. These p53-regulated gene products either reinforce or inhibit the p53 response and network. This then results in a coordination of cellular processes that need to be explored in future experiments.

\section{The cellular outputs of the downstream events}

This brief review has discussed at least six different outputs of the p53 pathway which fall into two distinct categories, primary response and secondary responses. The three primary responses to a stress input signal by the p53 pathway are; either cell cycle arrest, cellular senescence or apoptosis. The cell cycle arrest may be reversible or in other cases irreversible (which might be related to senescence). At this time we do not know all of the gene products ( $\mathrm{p} 53$ regulated or not) that bring about cell cycle arrest. While p21 clearly plays some role in a G-1 arrest, it is not the entire story (based upon knockout experiments). ${ }^{57,58}$ There is some controversy about whether p53 mediates a G-2 arrest, when it does so and how? There are several examples of a role for p53 in blocking the reinitiation of a second S-phase in cells that can not enter cytokinesis because of treatments with spindle poisons. ${ }^{59}$ This is one of the ways in which p53 prevents karyotypic instability in cells, which is a major function or output of the p53 pathway. Whether the p53 pathway has a method to 'count' the number of chromosomes that segregate into a daughter cell, and if this number is not correct it kills the cell, or it monitors segregation in another fashion remains an important line of enquiry? There is good evidence that the p53 protein and its functions regulate or monitor the number of centrosomes produced at each cell cycle. The p53 knockout mouse produces cells with abnormal numbers of centrosomes. ${ }^{60}$ The way in which the p53 pathway brings about cellular senescence is completely unknown. Just which p53-regulated genes initiate this state remain unclear. Indeed, there are very few reliable biomarkers for the senescent state and we do not know what mediates this process of irreversible division leaving the cell in a metabolically active state? There are good reasons to suspect that senescence occurs in vivo as well as in cell culture (cells from older animals undergo fewer divisions in culture) and plays a role in cancer responses. As such this is clearly an important line of research for the future. It appears that the majority of the p53-regulated genes play a role in promoting apoptosis. This pathway seems to contain examples of redundant functions so experiments where genes are knocked out are less informative. It also appears that tissue specific p53-regulated gene products play a role here. The transcriptional program that initiates apoptosis triggers both the intrinsic and extrinsic apoptotic pathways. The proposed p53 transcription-independent 
pathway to apoptosis needs additional evidence and several lines of proof that it is employed in vivo, and not only in cell culture under abnormal circumstances. It is an important potential mechanism and proof of its validity should be held to a high standard. An important question that remains in examining the output of the p53 pathway is how a cell which has received a stress signal chooses which of these three outputs (apoptosis, cell cycle arrest or cellular senescence) to implement? We do know that normal fibroblasts taken from an animal and irradiated undergo G-1 arrest while their transformed counterparts undergo apoptosis. Normal irradiated T cells undergo apoptosis, so both the state of cellular differentiation and the input of other signal transduction pathways influence this decision. In other experimental studies, hormones or other mediators which turn on signal transduction pathways altered the p53 output response. Now we need to understand the rules which regulate the p53-mediated output.

The secondary outputs of the p53 pathway come from p53regulated gene products that either (1) prevent DNA damage (sestrins) or aid in DNA repair, (2) mediate communication between the cell under stress and its neighbors, the extracellular matrix shared by these cells or even other more distant cells in the body, and (3) create intracellular or extracellular p53 feedback loops that modulate p53 activity and the pathway and simultaneously communicate with other networks in the cell. ${ }^{12}$ Prevention of DNA damage by removing free radicals or other chemical insults needs to be explored in more detail. This concept brings up the question of whether the $\mathrm{p} 53$ pathway is solely inductive, responding to stress and then preventing more damage, or is also active before a stress signal and is therefore truly preventing the initiation of DNA damage? The p53 functions that aid in DNA repair suggest that the cell cycle arrest that is preventing errors is reversible and the cell lives to divide again. The nature of the communication between a cell undergoing a p53 response (apoptosis, senescence or cell cycle arrest) and its neighbors is a poorly explored area of research. The soluble secreted p53-responsive proteins can change the extracellular matrix (maspin, PAI-1), block hormonal signaling (IGFBP3) to another pathway, and alter angiogenesis in the vicinity of the alarmed cells (thrombospondin). In addition p53 activation can enhance the rate of exosome production by the damaged cell by inducing the p53-regulated TSAP-6 gene. ${ }^{56,61}$ Exosomes can communicate with adjacent cells by cell fusion and with $T$ cells by presenting antigens to the immune system. Could p53-mediated apoptosis enhance immunization of the host in this fashion? The activation of p53 (and its inactivation) was first detected in virus-infected cells. ${ }^{1,2}$ Could the p53 pathway be playing an important role in infectious diseases and immunity? Is it possible that a cancer cell undergoing a p53 response is also immunizing its host to cancer antigens and preventing or slowing the disease in this fashion? If this was the case then cancer cells with mutant p53 would fail to immunize their host and selection for a mutant p53 could be due to an immunoselective process. These are provocative ideas that will need to be tested in the future. The negative- and positive-feedback loops that are initiated by p53-regulated genes and then act upon p53 activity and functions have been reviewed elsewhere ${ }^{12}$ and in this chapter. They serve two functions; (1) to modulate up or down p53 activity; and (2) to communicate with other signal transduction pathways in the cell. For example, after a commitment to apoptosis, a PTEN-mediated feedback loop modulates up p53 activity (a positive feedback) and shuts down the IGF-1 and mTOR pathways (growth and cell division pathways). This activates other processes such as autophagy which provides nutrients for adjacent cells and exosome production. Both autophagy and exosome production are functions of the lysosomal compartment and pathway. Could this indicate some additional fundamental roles of this cellular compartment in the p53 pathway and its control of cellular processes?

\section{Questions about the p53 pathway in a larger context}

Several observations have demonstrated that the p53 gene and its protein play a central role in the origins of cancers: (1) the role of the p53 protein and its gene in viral and cellular transformation, (2) the cancers in the p53 gene knockout mice, (3) cancers observed with the Li-Fraumeni families and (4) the high incidence of p53 gene mutations in many human cancers. However, just what does p53 do to prevent cancers from arising in murine and human populations? The presence of hundreds of genes in the p53 pathway and genetic polymorphisms in those genes suggests that individuals may have more or less efficient p53 pathways. The p53 gene itself has a codon 72 polymorphism (proline-arginine change) and the arginine allele appears to be more efficient in apoptosis in cell culture. ${ }^{62}$ The observation that the ratio of proline to arginine alleles in individuals changes as the latitude changes from the equator to the north pole already suggests that selection is playing a role upon these alleles. ${ }^{63}$ There are several studies that suggest that one or the other allele could influence the occurrence or progression of cancer. ${ }^{64,65} \mathrm{At}$ present however the results are confusing and a better understanding of the role of these two alleles in the pathway will be important. Recently, a polymorphism in the promoter of the human MDM-2 gene has been identified (SNP309). ${ }^{66}$ The minor allele enhances the levels of the MDM-2 protein in cells, lowers the levels of p53 after a stress response and lowers the frequency of apoptosis in these cells. This same allele lowers the age of onset of the development of several types of cancer. ${ }^{66}$ More recently, a separate study demonstrated that the minor allele of SNP309 increased the frequency of esophageal cancer in individuals and that the codon 72 polymorphism further increased the odds ratio of developing this cancer (in an additive fashion) and that smoking further increased this odds ratio (in a more than additive fashion). ${ }^{67}$ Clearly this is one way to relate the participation of the p53 pathway to the development of cancers, the progression of cancers and the treatment of cancers. There will be more p53 pathway polymorphisms, more environmental variables tested and more studies that will uncover the functions of the p53 network in humans.

Several lines of evidence suggest that the p53 pathway contains a sexual dimorphism that distinguishes gender responses. The p53 gene knockout mice have a sex ratio distortion, the severity of which depends upon the genetic 
background of the inbred strain. ${ }^{68-70}$ This is due to the fact that many female mice are born with an exencephalic condition, where the brain case never closes and the brain is outside of the head. ${ }^{69}$ This is seen only in females. Whether the cranium fails to close or be developed or the brain is too large (failure of neuronal apoptosis in development?) remains unclear and will be important to explore. The genetic background determines the penetrance of this phenotype so some female mice are born. Female heterozygous p53 knockout mice develop more osteogenic sarcomas than do their male counterparts indicating that sexual dimorphism is operative. ${ }^{71,72}$ In humans with Li-Fraumeni Syndrome (also heterozygotes) the females develop cancers at a higher frequency and earlier in life ${ }^{73}$ and this is the case even if breast and prostate cancers are not considered. The MDM-2 gene, can under certain circumstances be regulated by the estrogen receptor. $^{74}$ The IGF-1 pathway (which interfaces with the p53 pathway via the activation of MDM-2 by AKT-1) is also influenced by sexual hormones and sensory inputs. ${ }^{39,40}$ The SIR-2 or SIRT-1 gene product, an NAD-dependent histone deacetylase, acts to remove acetyl groups from both the forkhead and the p53 proteins and can modulate the activity of the IGF-1 pathway. ${ }^{75,76}$ Certain types of mutations in the IGF-1 pathway and SIR2/SIRT-1 play a central role in extending the longevity of an organism (yeast, worms, flies and mice). ${ }^{77,78}$ Will the $\mathrm{p} 53$ pathway play a role in determining longevity in worms, flies, mice and humans? Longevity is also sexually dimorphic in some of these species. In mice (2-3 years life span) and dogs (8-16 years life span) and humans (75 years life span) most cancers arise in the last quarter of their life spans rather than a specific number of years after birth. In fact the frequency of cancer in these species increases exponentially (over 1 - to 10000 -fold) during the last quarter of their life span. Thus evolutionary selection has fixed in each of these species the age of sexual maturity, the age of producing offspring, the age of developing the majority of cancers in the population, and in relation to that, the longevity of the species (there would be no species if you died before reproducing). The longevity of a species may be set in part by alterations in the IGF-1 pathway and the age of onset of cancers by tumor suppressor genes like p53 and their pathways. The reproductive age is fixed by a related and interactive set of genes and their networks. Future experiments will explore these variables and their relationships.

In this evolutionary context, how did a gene function like p53 arise and what does (did) it do for the animal? Worms and flies are born with a body plan that is largely (except for the germ line) postmitotic. No more divisions occur in an adult animal. In these cases the major job of p53 is the surveillance of the germ line for environmental insults that would result in poor offspring. The body plan of adult vertebrates, however, contains many tissues that continue to divide throughout life and renew themselves. Here p53 has been employed in the surveillance of somatic cells to prevent cancers from arising in the organism. The exploration of the functions of the p53 pathway throughout evolution and how it has adapted to novel situations and organisms will be a profitable avenue of research.

We now know enough about the p53 pathway to move in some new directions. One of the input signals that activates p53 is nitric oxide which is produced and employed for signaling after inflammatory responses. Indeed inflammatory syndromes have been shown to cause cells in the vicinity of lymphocyte invasions to undergo a p53 response. ${ }^{79,80}$ Communication may occur in the other direction with p53regulated exosomes signaling to $T$ cells and immunizing the organism with parts of damaged cells. These ideas suggest the exploration of whether p53 plays a role in the recovery from infectious diseases, inflammation or the initiation of autoimmunity or the establishment of tolerance? The apoptotic functions of the $\mathrm{p} 53$ pathway may normally be employed by the immune system for optimal function. These same apoptotic pathways could play a role in neurodegenerative diseases. There is a growing body of evidence for a p53regulated program cell death in neurons, glial cells or Schwann cells. What role does the p53 pathway and its response to environmental insults play in the origins or propagation of neurodegenerative diseases? With an increasing age of the host does the p53 pathway become more active (is there a pathological condition that makes it more active?) or does it decay in its efficiency? Could it be the case that the large and rapid increase in cancers in a population during the last quarter of our life span is due to accumulated mutations in oncogenes and tumor suppressor genes in a cell and a declining efficiency of the p53 response which increases the rate of these fixed mutations in that cell? These are more complicated questions to answer and more difficult experiments to perform but the answers will open up new avenues of research and understanding.

One thing we can be sure of, some of the answers to the questions posed in this chapter will be answered. Other questions will be rephrased or changed completely before it is productive to test these ideas. However, all of these questions will be discussed, probed and eventually explored. We have uncovered and explored a process central to life, how a cell responds to stress or perturbations in its environment. Understanding these homeostatic mechanisms central to all life processes ensures a productive future of questioning.

\section{References}

1. Lane DP and Crawford LV (1979) T antigen is bound to a host protein in SV40transformed cells. Nature 278: 261-263.

2. Linzer DI and Levine AJ (1979) Characterization of a $54 \mathrm{~K}$ dalton cellular SV40 tumor antigen present in SV40-transformed cells and uninfected embryonal carcinoma cells. Cell 17: 43-52.

3. Kress M, May E, Cassingena R and May P (1979) Simian virus 40-transformed cells express new species of proteins precipitable by anti-simian virus 40 tumor serum. J. Virol. 31: 472-483.

4. Deleo AB, Jay G, Appella E, Dubois GC, Law LW and Old LJ (1979) Detection of a transformation-related antigen in chemically induced sarcomas and other transformed cells of the mouse. Proc. Natl. Acad. Sci. USA 76: 2420-2424.

5. Levine AJ, Finlay CA and Hinds PW (2004) P53 is a tumor suppressor gene. Cell 116 (2 Suppl): S67-S69 1 p following S69.

6. Robins H, Alexe G, Harris SL and Levine AJ (2005) The first twenty-five years of p53 research In 25 Years of p53 Research Hainaut P, Wiman KG (eds) Dordrecht: Springer pp. 1-26.

7. Vogelstein B, Lane D and Levine AJ (2000) Surfing the p53 network. Nature 408: 307-310.

8. Giaccia AJ and Kastan MB (1998) The complexity of p53 modulation: emerging patterns from divergent signals. Genes Dev. 12: 2973-2983. 
9. Gudkov AV and Komarova EA (2003) The role of p53 in determining sensitivity to radiotherapy. Nat. Rev. Cancer 3: 117-129.

10. Oren M (2003) Decision making by p53: life, death and cancer. Cell Death Differ. 10: 431-442.

11. Soussi $T$ (2005) The p53 pathway and human cancer. Br. J. Surg. 92: 1331-1332.

12. Harris SL and Levine AJ (2005) The p53 pathway: positive and negative feedback loops. Oncogene 24: 2899-2908.

13. Feng Z, Zhang H, Levine AJ and Jin S (2005) The coordinate regulation of the p53 and mTOR pathways in cells. Proc. Natl. Acad. Sci. USA 102: 8204-8209.

14. Jones RG, Plas DR, Kubek S, Buzzai M, Mu J, Xu Y, Birnbaum MJ and Thompson CB (2005) AMP-activated protein kinase induces a p53-dependent metabolic checkpoint. Mol. Cell 18: 283-293.

15. Yang A, Kaghad M, Caput $D$ and McKeon $F$ (2002) On the shoulders of giants: p63, p73 and the rise of p53. Trends Genet. 18: 90-95.

16. McKeon $F$ and Yang $A$ (2005) p53, p63, and p73: internecine relations? In 25 Years of p53 Research Hainaut P, Wiman KG (eds) Dordrecht: Springer pp. 209-222.

17. Monti O, Damalas A, Strano S and Blandino G (2005) p73, p63 and mutant p53: members of protein complexs floating in cancer cells In 25 Years for p53 Research Hainaut P, Wiman KG (eds) Dordrecht: Springer pp. 223-232.

18. Hoh J, Jin S, Parrado T, Edington J, Levine AJ and Ott J (2002) The p53MH algorithm and its application in detecting p53-responsive genes. Proc. Natl. Acad. Sci. USA 99: 8467-8472.

19. Leng RP, Lin Y, Ma W, Wu H, Lemmers B, Chung S, Parant JM, Lozano G, Hakem R and Benchimol S (2003) Pirh2, a p53-induced ubiquitin-protein ligase, promotes p53 degradation. Cell 112: 779-791.

20. Dornan D, Wertz I, Shimizu H, Arnott D, Frantz GD, Dowd P, O'Rourke K Koeppen $\mathrm{H}$ and Dixit VM (2004) The ubiquitin ligase COP1 is a critical negative regulator of p53. Nature 429: 86-92.

21. Stommel JM and Wahl GM (2004) Accelerated MDM2 auto-degradation induced by DNA-damage kinases is required for p53 activation. EMBO J. 23: 1547-1556.

22. Appella E and Anderson CW (2001) Post-translational modifications and activation of p53 by genotoxic stresses. Eur. J. Biochem. 268: 2764-2772.

23. Xirodimas DP, Saville MK, Bourdon JC, Hay RT and Lane DP (2004) Mdm2 mediated NEDD8 conjugation of p53 inhibits its transcriptional activity. Cell 118 83-97.

24. Brooks CL and Gu W (2003) Ubiquitination, phosphorylation and acetylation: the molecular basis for p53 regulation. Curr. Opin. Cell Biol. 15: 164-171.

25. Xu Y (2003) Regulation of p53 responses by post-translational modifications. Cell Death Differ. 10: 400-403.

26. Vassilev LT, Vu BT, Graves B, Carvajal D, Podlaski F, Filipovic Z, Kong N, Kammlott U, Lukacs C, Klein C, Fotouhi N and Liu EA (2004) In vivo activation of the p53 pathway by small-molecule antagonists of MDM2. Science 303 : $844-848$.

27. McKinney K, Mattia M, Gottifredi V and Prives C (2004) p53 linear diffusion along DNA requires its $C$ terminus. Mol. Cell 16: 413-424.

28. McKinney K and Prives $C$ (2005) Regulation of p53 DNA binding In 25 Years of p53 Research Hainaut P, Wiman KG (eds) Dordrecht: Springer pp. 27-52.

29. Lowe SW and Sherr CJ (2003) Tumor suppression by Ink4a-Arf: progress and puzzles. Curr. Opin. Genet. Dev. 13: 77-83.

30. Hemann MT and Lowe S (2005) p53 links tumor development to cancer therapy In 25 Years of $p 53$ Research Hainaut P, Wiman KG (eds) Dordrecht: Springer pp. 339-352.

31. Lev Bar-Or R, Maya R, Segel LA, Alon U, Levine AJ and Oren M (2000) Generation of oscillations by the p53-Mdm2 feedback loop: a theoretical and experimental study. Proc. Natl. Acad. Sci. USA 97: 11250-11255.

32. Lahav G, Rosenfeld N, Sigal A, Geva-Zatorsky N, Levine AJ, Elowitz MB and Alon U (2004) Dynamics of the p53-Mdm2 feedback loop in individual cells. Nat. Genet. 36: 147-150.

33. Momand J, Zambetti GP, Olson DC, George D and Levine AJ (1992) The $\mathrm{mdm}-2$ oncogene product forms a complex with the p53 protein and inhibits p53-mediated transactivation. Cell 69: 1237-1245.

34. Oliner JD, Kinzler KW, Meltzer PS, George DL and Vogelstein B (1992) Amplification of a gene encoding a p53-associated protein in human sarcomas. Nature 358: 80-83

35. Montes de Oca Luna R, Wagner DS and Lozano G (1995) Rescue of early embryonic lethality in mdm2-deficient mice by deletion of p53. Nature 378 : 203-206.
36. Parant J, Chavez-Reyes A, Little NA, Yan W, Reinke V, Jochemsen AG and Lozano G (2001) Rescue of embryonic lethality in Mdm4-null mice by loss of Trp53 suggests a nonoverlapping pathway with MDM2 to regulate p53. Nat. Genet. 29: 92-95.

37. Tao W and Levine AJ (1999) Nucleocytoplasmic shuttling of oncoprotein Hdm2 is required for Hdm2-mediated degradation of p53. Proc. Natl. Acad. Sci. USA 96: 3077-3080.

38. Boyd SD, Tsai KY and Jacks T (2000) An intact HDM2 RING-finger domain is required for nuclear exclusion of p53. Nat. Cell Biol. 2: 563-568.

39. Mayo LD and Donner DB (2001) A phosphatidylinositol 3-kinase/Akt pathway promotes translocation of Mdm2 from the cytoplasm to the nucleus. Proc. Natl. Acad. Sci. USA 98: 11598-11603.

40. Zhou BP, Liao Y, Xia W, Zou Y, Spohn B and Hung MC (2001) HER-2/neu induces p53 ubiquitination via Akt-mediated MDM2 phosphorylation. Nat. Cell Biol. 3: 973-982

41. Okamoto K, Li H, Jensen MR, Zhang T, Taya $Y$, Thorgeirsson SS and Prives C (2002) Cyclin G recruits PP2A to dephosphorylate Mdm2. Mol. Cell 9: 761-771.

42. Levine AJ, Feng Z, Mak T, You H and Jin S (2006) Coordination and communication between the p53 and IGF-1-AKT-TOR signal transduction pathways. Genes Dev. 20: 267-275.

43. Bourdon JC, Fernandes K, Murray-Zmijewski F, Liu G, Diot A, Xirodimas DP, Saville MK and Lane DP (2005) p53 isoforms can regulate p53 transcriptional activity. Genes Dev. 19: 2122-2137.

44. Chen J, Ruan H, Ng SM, Gao C, Soo HM, Wu W, Zhang Z, Wen Z, Lane DP and Peng $J(2005)$ Loss of function of def selectively up-regulates ।\{Deltal\}113p53 expression to arrest expansion growth of digestive organs in zebrafish. Genes Dev. 19: 2900-2911.

45. el-Deiry WS, Kern SE, Pietenpol JA, Kinzler KW and Vogelstein B (1992) Definition of a consensus binding site for p53. Nat. Genet. 1: 45-49.

46. Feng Z, Jin S, Zupnick A, Hoh J, de Stanchina E, Lowe S, , Prives C and Levine AJ (2005) p53 tumor suppressor protein regulates the levels of huntingtin gene expression. Oncogene 24: 1-7.

47. Zhao R, Gish K, Murphy M, Yin Y, Notterman D, Hoffman WH, Tom E, Mack DH and Levine AJ (2000) The transcriptional program following p53 activation. Cold Spring Harb. Symp. Quant. Biol. 65: 475-482.

48. Moll UM, Wolff S, Speidel D and Deppert W (2005) Transcription-independent pro-apoptotic functions of p53. Curr. Opin. Cell Biol. 17: 631-636.

49. Chipuk JE, Kuwana T, Bouchier-Hayes L, Droin NM, Newmeyer DD, Schuler M and Green DR (2004) Direct activation of Bax by p53 mediates mitochondrial membrane permeabilization and apoptosis. Science 303: 1010-1014

50. Donehower LA (2002) Does p53 affect organismal aging? J. Cell Physiol. 192: 23-33.

51. Budanov AV, Sablina AA, Feinstein E, Koonin EV and Chumakov PM (2004) Regeneration of peroxiredoxins by p53-regulated sestrins, homologs of bacterial AhpD. Science 304: 596-600.

52. Buckbinder L, Talbott R, Velasco-Miguel S, Takenaka I, Faha B, Seizinger BR and Kley N (1995) Induction of the growth inhibitor IGF-binding protein 3 by p53. Nature 377: 646-649.

53. Kunz C, Pebler S, Otte J and von der Ahe D (1995) Differential regulation of plasminogen activator and inhibitor gene transcription by the tumor suppressor p53. Nucl. Acids Res. 23: 3710-3717.

54. Zou Z, Gao C, Nagaich AK, Connell T, Saito S, Moul JW, Seth P, Appella E and Srivastava $S(2000)$ p53 regulates the expression of the tumor suppressor gene maspin. J. Biol. Chem. 275: 6051-6054.

55. Dameron KM, Volpert OV, Tainsky MA and Bouck N (1994) Control of angiogenesis in fibroblasts by p53 regulation of thrombospondin-1. Science 265: 1582-1584

56. Amzallag N, Passer BJ, Allanic D, Segura E, Thery C, Goud B, Amson R and Telerman A (2004) TSAP6 facilitates the secretion of translationally controlled tumor protein/histamine-releasing factor via a nonclassical pathway. J. Biol. Chem. 279: 46104-46112.

57. Deng C, Zhang P, Harper JW, Elledge SJ and Leder P (1995) Mice lacking p21CIP1/WAF1 undergo normal development, but are defective in G1 checkpoint control. Cell 82: 675-684.

58. Brugarolas J, Chandrasekaran C, Gordon JI, Beach D, Jacks T and Hannon GJ (1995) Radiation-induced cell cycle arrest compromised by p21 deficiency. Nature 377: 552-557. 
59. Vaziri C, Saxena S, Jeon Y, Lee C, Murata K, Machida Y, Wagle N, Hwang DS and Dutta A (2003) A p53-dependent checkpoint pathway prevents rereplication. Mol. Cell 11: 997-1008.

60. Wang XJ, Greenhalgh DA, Jiang A, He D, Zhong L, Brinkley BR and Roop DR (1998) Analysis of centrosome abnormalities and angiogenesis in epidermaltargeted p53172 $\mathrm{H}$ mutant and p53-knockout mice after chemical carcinogenesis: evidence for a gain of function. Mol. Carcinog. 23: 185-192.

61. Passer BJ, Nancy-Portebois V, Amzallag N, Prieur S, Cans C, Roborel de Climens A, Fiucci G, Bouvard V, Tuynder M, Susini L, Morchoisne S, Crible V, Lespagnol A, Dausset J, Oren M, Amson R and Telerman A (2003) The p53-inducible TSAP6 gene product regulates apoptosis and the cell cycle and interacts with Nix and the Myt1 kinase. Proc. Natl. Acad. Sci. USA 100: 2284-2289.

62. Dumont P, Leu JI, Della Pietra III AC, George DL and Murphy M (2003) The codon 72 polymorphic variants of p53 have markedly different apoptotic potential. Nat. Genet. 33: 357-365.

63. Khrunin AV, Tarskaia LA, Spitsyn VA, Lylova OI, Bebyakova NA, Mikulich A and Limborska SA (2005) p53 polymorphisms in Russia and Belarus: correlation of the 2-1-1 haplotype frequency with longitude. Mol. Genet. Genomics 272: 666-672.

64. Ohayon T, Gershoni-Baruch R, Papa MZ, Distelman Menachem T, Eisenberg Barzilai S and Friedman E (2005) The R72P P53 mutation is associated with familial breast cancer in Jewish women. Br. J. Cancer 92: 1144-1148.

65. van Heemst D, Mooijaart SP, Beekman M, Schreuder J, de Craen AJ, Brandt BW, Slagboom PE and Westendorp RG (2005) Variation in the human TP53 gene affects old age survival and cancer mortality. Exp. Gerontol. 40: 11-15.

66. Bond GL, Hu W, Bond EE, Robins H, Lutzker SG, Arva NC, Bargonetti J, Barte F, Taubert H, Wuerl P, Onel K, Yip L, Hwang SJ, Strong LC, Lozano G and Levine AJ (2004) A single nucleotide polymorphism in the MDM2 promoter attenuates the p53 tumor suppressor pathway and accelerates tumor formation in humans. Cell 119: 591-602.

67. Hong Y, Miao X, Zhang X, Ding F, Luo A, Guo Y, Tan W, Liu Z and Lin D (2005) The role of $\mathrm{P} 53$ and MDM2 polymorphisms in the risk of esophageal squamous cell carcinoma. Cancer Res. 65: 9582-9587.

68. Rotter V, Schwartz D, Almon E, Goldfinger N, Kapon A, Meshorer A, Donehower LA and Levine AJ (1993) Mice with reduced levels of p53 protein exhibit the testicular giant-cell degenerative syndrome. Proc. Natl. Acad. Sci. USA 90: 9075-9079.
69. Sah VP, Attardi LD, Mulligan GJ, Williams BO, Bronson RT and Jacks T (1995) A subset of p53-deficient embryos exhibit exencephaly. Nat. Genet. 10: $175-180$.

70. Armstrong JF, Kaufman MH, Harrison DJ and Clarke AR (1995) Highfrequency developmental abnormalities in p53-deficient mice. Curr. Biol. 5: 931-936.

71. Mitsumori K, Shimo T, Onodera H, Takagi H, Yasuhara K, Tamura T, Aoki Y, Nagata $O$ and Hirose M (2000) Modifying effects of ethinylestradiol but not methoxychlor on $\mathrm{N}$-ethyl- $\mathrm{N}$-nitrosourea-induced uterine carcinogenesis in heterozygous p53-deficient CBA mice. Toxicol. Sci. 58: 43-49.

72. Baatout S, Jacquet P, Michaux A, Buset J, Vankerkom J, Derradji H, Yan J, Von Suchodoletz H, De Saint-Georges L, Desaintes C and Mergeay M (2002) Developmental abnormalities induced by X-irradiation in p53 deficient mice. In Vivo 16: 215-221.

73. Strong LC (2003) General keynote: hereditary cancer: lessons from Li-Fraumeni syndrome. Gynecol. Oncol. 88 (1 Part 2): S4-S7; discussion S11-S13.

74. Kinyamu HK and Archer TK (2003) Estrogen receptor-dependent proteasoma degradation of the glucocorticoid receptor is coupled to an increase in $\mathrm{mdm} 2$ protein expression. Mol. Cell. Biol. 23: 5867-5881.

75. Luo J, Nikolaev AY, Imai S, Chen D, Su F, Shiloh A, Guarente L and Gu W (2001) Negative control of p53 by Sir2alpha promotes cell survival under stress. Cell 107: 137-148.

76. Motta MC, Divecha N, Lemieux M, Kamel C, Chen D, Gu W, Bultsma Y, McBurney M and Guarente L (2004) Mammalian SIRT1 represses forkhead transcription factors. Cell 116: 551-563.

77. Kenyon C (2005) The plasticity of aging: insights from long-lived mutants. Cell 120: 449-460.

78. Guarente $L$ and Picard F (2005) Calorie restriction - the SIR2 connection. Cell 120: $473-482$.

79. Hofseth LJ, Saito S, Hussain SP, Espey MG, Miranda KM, Araki Y, Jhappan C, Higashimoto Y, He P, Linke SP, Quezado MM, Zurer I, Rotter V, Wink DA, Appella E and Harris CC (2003) Nitric oxide-induced cellular stress and p53 activation in chronic inflammation. Proc. Natl. Acad. Sci. USA 100: 143-148.

80. Valenti LM, Mathieu J, Chancerelle Y, De Sousa M, Levacher M, Dinh-Xuan AT and Florentin I (2005) High levels of endogenous nitric oxide produced after burn injury in rats arrest activated Tlymphocytes in the first G1 phase of the cell cycle and then induce their apoptosis. Exp. Cell Res. 306: 150-167. 